\title{
Elemental Chalcogen (Se, $S$ ) in PEG-400 to the Synthesis of Seleno- and Thioflavones from 2-Chlorophenyl Ethynyl Ketone and Nucleophilic Species of Chalcogen
}

\author{
Patrick C. Nobre, ${ }^{a}$ Thiago J. Peglow, ${ }^{a}$ Ricardo H. Bartz, ${ }^{a}$ Angelita M. Barcellos, ${ }^{\oplus a}$ \\ Raquel G. Jacob, ${ }^{a}$ Márcio S. Silva, ${ }^{a}$ Thiago Barcellos ${ }^{\circledR} *, b$ and Gelson Perin ${ }^{\oplus *, a}$
}

${ }^{a}$ Laboratório de Síntese Orgânica Limpa (LASOL), Centro de Ciências Químicas, Farmacêuticas e de Alimentos (CCQFA), Universidade Federal de Pelotas (UFPel), PO Box 354, 96010-900 Pelotas-RS, Brazil

${ }^{b}$ Laboratório de Biotecnologia de Produtos Naturais e Sintéticos, Universidade de Caxias do Sul (UCS), 95070-560 Caxias do Sul-RS, Brazil

\begin{abstract}
An alternative green method was developed for the synthesis of thio- and selenoflavones by the ring closure of 2-chlorophenyl ethynyl ketone with $\mathrm{NaHY}(\mathrm{Y}=\mathrm{S}, \mathrm{Se})$. These nucleophilic chalcogen species were generated in situ using $\mathrm{NaBH}_{4}$ to reduce the elemental chalcogen in the presence of polyethylene glycol-400 (PEG-400). The efficiency of this reaction is strongly dependent on the PEG-400 solvent, acting like a crown ether, complexing with the sodium atom of $\mathrm{NaHY}$ species, making the chalcogen nucleophile more active. The synthetic protocol proceeded efficiently at $100{ }^{\circ} \mathrm{C}$ under argon, using a range of 2-chlorophenyl ethynyl ketone containing alkyl, aryl, or vinyl groups and the sulfur and selenium chalcogen. By this efficient and simple approach, 18 chalcogenoflavones were obtained in good to excellent yields after $2 \mathrm{~h}$.
\end{abstract}

Keywords: chromone, green chemistry, polyethyelene glycol-400, selenium, sulfur

\section{Introduction}

Interest in organochalcogen compounds has been continuously increasing due to their reputable synthetic and biologic applications. ${ }^{1}$ In this field, the modification of heterocyclic compounds with sulfur, selenium, and tellurium atom is a good strategy to obtain more active species and increase the biological applicability. ${ }^{2}$ Among the several heterocyclic cores, flavones and chromone derivatives constitute part of a large family of natural products with pharmaceutical applications. The flavone system (2-phenyl$4 H$-chromen-4-one, shown in Figure 1) is a key structural which play important roles in numerous biological processes. ${ }^{3}$ Replacement of the oxygen atom in the pyran ring with selenium and sulfur results in selenoflavone and thioflavone, respectively (Figure 1). The chalcogenoflavones have received intensive attention from medicinal chemists due to their unique structure-activity relationship showing antioxidant, anti-inflammatory, anti-cancer, antimicrobial, and antifungal activities, among others. ${ }^{4}$

Regarding the synthesis of these bioactive compounds,

*e-mail: thiago.barcellos@ucs.br; gelson_perin@ufpel.edu.br
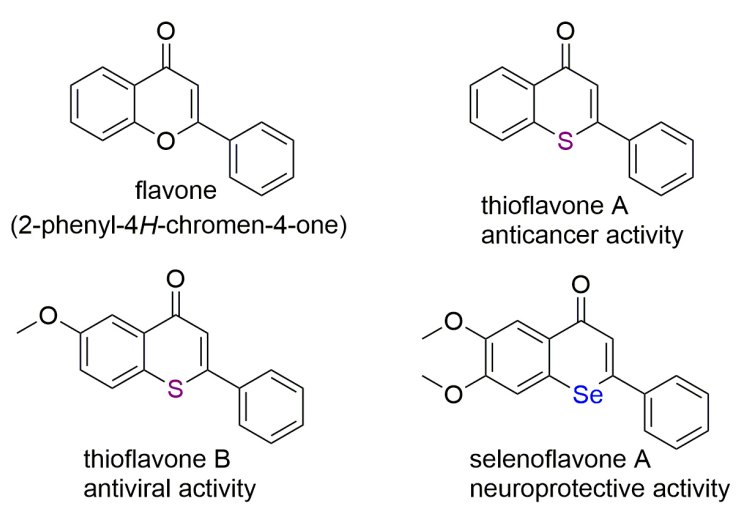

Figure 1. The flavone structure and its derivatives.

they can be prepared by two main procedures: intramolecular cyclization of 2-alkylchalcogenolated alkynones; ${ }^{5}$ and ring closure of 2-halophenyl ethynyl ketones with $-\mathrm{SeH}$ or $-\mathrm{SH}$ moiety. ${ }^{6}$ Also, the thioflavone was prepared using non-conventional methodologies, such as multicomponent reactions, ${ }^{7}$ intramolecular cyclization ${ }^{8}$ and rearrangement, ${ }^{9}$ decarbonylative cycloaddition, ${ }^{10}$ condensation, ${ }^{11}$ among others. ${ }^{12}$ However, greener synthetic methods for the synthesis of both selenoflavones and thioflavones are still limited. 
In the context of green chemistry, several synthetic approaches have been progressed, producing a wide variety of compounds without or less environmental hazard. ${ }^{13}$ A pivotal parameter to improve the sustainability of a reaction is the use of a suitable medium for environmentally friendly and safe chemical reactions. ${ }^{14}$ Thus, polyethylene glycol-400 (PEG-400) might be a solvent with higher preference because of its versatile blessings as a green reaction media. PEG solvent is perceived to be cheap, easily accessible, non-volatile, biodegradable, biocompatible, with low flammability, recyclable, and thermally stable..$^{14,15}$

Noticeable organic transformations involving PEG as a green reaction media were reported. ${ }^{16}$ Specifically, this solvent has been described as the reaction medium for procedures involving organochalcogen compounds. ${ }^{17}$ Our research group has recently described the in situ generation of chalcogenolate anions using the system RYYR/NaBH$/$ PEG-400 ( $\mathrm{Y}=\mathrm{S}, \mathrm{Se}$, and Te) and their use in selective reactions..$^{18}$ So far, only two procedures were described using $\mathrm{NaBH}_{4} / \mathrm{PEG}-400$ system to reduce elemental chalcogen and their application to prepare bis(2-pyridyl) diselenide derivatives and divinyl selenides/tellurides. ${ }^{19}$ We hypothesized that the nucleophilic chalcogen species generated in situ could be applied in other transformations. To test this conjecture and develop our continued interest in green chemistry, we herein disclose our latest work on the synthesis of selenoflavones $\mathbf{3}(\mathrm{Y}=\mathrm{Se})$ and thioflavones 4 $(\mathrm{Y}=\mathrm{S}$ ) by ring closure of 2-chlorophenyl ethynyl ketone $\mathbf{2}$ under mild conditions (Scheme 1).

\section{Experimental}

\section{General information}

All reagents and solvents used were purchased from commercial sources (Sigma-Aldrich $^{\circledR}$, São Paulo, Brazil). The reactions were monitored by thin-layer chromatography (TLC) carried out on Merk silica gel $\left(60 \mathrm{~F}_{254}\right)$ by using UV light as visualization agent and the mixture between $5 \%$ of vanillin in $10 \%$ of $\mathrm{H}_{2} \mathrm{SO}_{4}$ under heating conditions as developing agents. Merck silica gel (particle size 0.040-0.063 mm) was used to flash chromatography. Hydrogen nuclear magnetic resonance spectra ( ${ }^{1} \mathrm{H}$ NMR) were obtained on Bruker
Avance III HD 400 MHz (Uster, Switzerland) employing a direct broadband probe at $400 \mathrm{MHz}$. The spectra were recorded in $\mathrm{CDCl}_{3}$ solutions. The chemical shifts are reported in ppm, referenced to tetramethylsilane (TMS) as the internal reference. Coupling constants $(J)$ are reported in hertz. Abbreviations to denote the multiplicity of a particular signal are s (singlet), d (doublet), dd (doublet of doublet), $\mathrm{dt}$ (doublet of triplet), $\mathrm{t}$ (triplet), q (quartet), quint (quintet) and $\mathrm{m}$ (multiplet). Carbon-13 nuclear magnetic resonance spectra $\left({ }^{13} \mathrm{C}\right.$ NMR) were obtained on Bruker Avance III HD $400 \mathrm{MHz}$ (Uster, Switzerland) employing a direct broadband probe at $100 \mathrm{MHz}$. The chemical shifts are reported in $\mathrm{ppm}$, referenced to the solvent peak of $\mathrm{CDCl}_{3}(\delta 77.0 \mathrm{ppm})$. Selenium-77 nuclear magnetic resonance spectra ( ${ }^{77} \mathrm{Se}$ NMR) were obtained on Bruker Avance III HD $400 \mathrm{MHz}$ (Uster, Switzerland) employing a direct broadband probe at $76 \mathrm{MHz}$, measured without ${ }^{1} \mathrm{H}$ decoupling. The chemical shifts are reported in ppm, using as solvent the $\mathrm{CDCl}_{3}$ and as an internal standard the diphenyl diselenide ( $\delta 463.0 \mathrm{ppm}$ ). The high-resolution mass spectrometry (HRMS) quadrupole time-of-flight (QTOF) analyses were performed on a Bruker (Billerica, MA, USA) Daltonics micrOTOF-Q II instrument in positive mode. The samples were solubilized in high performance liquid chromatography (HPLC)-grade acetonitrile and injected into the atmospheric pressure chemical ionization (APCI) source by means of a syringe pump at a flow rate of $5.0 \mu \mathrm{L} \mathrm{min}$. The follow instrument parameters were applied: capillary and cone voltages were set to +3500 and $-500 \mathrm{~V}$, respectively, with a desolvation temperature of $180^{\circ} \mathrm{C}$. For data acquisition and processing, Compass 1.3 for micrOTOF-Q II software (Bruker Daltonics, USA) was used. The data were collected in the $\mathrm{m} / \mathrm{z}$ range of 50-1200 at the speed of two scans per s. Low-resolution mass spectra were obtained with a Shimadzu (Kyoto, Japan) GC-MS-QP2010P mass spectrometer. Melting point (mp) values were measured in a Marte (São Paulo, Brazil) PFD III instrument with a $0.1^{\circ} \mathrm{C}$ precision.

General procedure for the synthesis of 2-chloroaryl ethynyl ketone 2

Procedure adapted from the literature..$^{20}$ To a solution of corresponding acetylene (5.0 mmol, 1.0 equiv) in

$$
\begin{aligned}
& Y^{0} \frac{\mathrm{PEG}-400, \mathrm{NaBH}_{4}}{\mathrm{Ar}, 50{ }^{\circ} \mathrm{C}, 0.5 \mathrm{~h}}\left[\begin{array}{c}
\mathrm{NaHY} \\
\text { "in situ" }
\end{array}\right. \\
& \mathrm{Y}^{0}=\mathrm{Se} \mathrm{1a} \\
& \mathrm{Y}^{0}=\mathrm{S} \mathbf{1 b} \\
& \mathrm{R}=\mathrm{H}, \mathrm{Cl} ; \mathrm{R}^{1}=\text { aryl, alkyl, vinyl }
\end{aligned}
$$

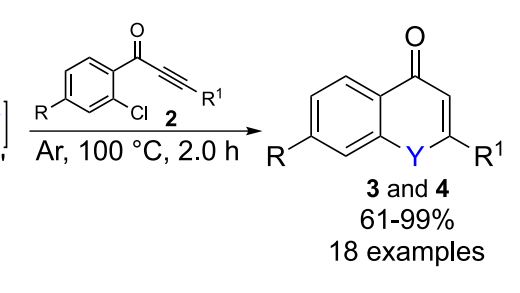

Scheme 1. PEG-400 mediate synthesis of the chalcogenoflavones. 
dry tetrahydrofuran (THF) $(25 \mathrm{~mL})$ was added ${ }^{n} \mathrm{BuLi}$ (2.5 mol L ${ }^{-1}$ in ${ }^{n}$ hexane, $2.2 \mathrm{~mL}, 5.5 \mathrm{mmol}, 1.1$ equiv) dropwise at $-78{ }^{\circ} \mathrm{C}$ under nitrogen atmosphere. After the reaction mixture had been stirred at $-78^{\circ} \mathrm{C}$ for $1 \mathrm{~h}$, 2-chlorobenzaldehyde (5.0 mmol, 1.0 equiv) was added dropwise at $-78{ }^{\circ} \mathrm{C}$. Upon stirring at same temperature for $1 \mathrm{~h}$, the reaction solution was quenched with saturated solution of $\mathrm{NH}_{4} \mathrm{Cl}$ and extracted with ethyl acetate $(3 \times 20 \mathrm{~mL})$. The combined organic layer was dried over anhydrous $\mathrm{MgSO}_{4}$, filtered, and concentrated under reduced pressure to give the crude alcohol product. After, dichloromethane $(15 \mathrm{~mL})$ and $\mathrm{MnO}_{2}$ (10 equiv) were added and the solution was stirred at room temperature till alcohol disappeared by TLC analysis. The reaction solution was filtered over celite, solvents were removed, and the crude product was purified by column chromatography on silica gel with hexane/EtOAc and concentrated to afford the corresponding 2-chlorophenyl ethynyl ketone 2 .

General procedure for synthesis of chalcogenoflavones 3 and 4

In a $10.0 \mathrm{~mL}$ reaction vial containing Se or $\mathrm{S}(0.3 \mathrm{mmol})$ in PEG-400 (2.0 mL) under argon atmosphere was added $\mathrm{NaBH}_{4}(0.7 \mathrm{mmol})$, and the mixture was slowly heated to $50{ }^{\circ} \mathrm{C}$ being stirred for $30 \mathrm{~min}$. Then, compound 2 $(0.25 \mathrm{mmol})$ was added, and the temperature was raised to $100{ }^{\circ} \mathrm{C}$. The reaction mixture remained under magnetic stirring for $2 \mathrm{~h}$. Then, water was added $(25.0 \mathrm{~mL})$ and the reaction was extracted with ethyl acetate $(3 \times 10.0 \mathrm{~mL})$. The organic phase was separated, dried over $\mathrm{MgSO}_{4}$, and the solvent was evaporated under reduced pressure. The product $\mathbf{3}$ or $\mathbf{4}$ was isolated by column chromatography using hexane/ethyl acetate (95/5) as eluent.

\section{2-Phenyl-4H-selenochromen-4-one (3a) ${ }^{6}$}

Yield: $0.070 \mathrm{~g}(98 \%)$; yellowish solid; $\mathrm{mp} 123-125^{\circ} \mathrm{C}$; ${ }^{1} \mathrm{H}$ NMR $\left(400 \mathrm{MHz}, \mathrm{CDCl}_{3}\right) \delta 7.28$ (s, 1H, Ar-H), 7.35-7.46 (m, 5H, Ar-H), 7.50-7.54 (m, 2H, Ar-H), 7.57-7.59 (m, 1H, $\mathrm{Ar}-\mathrm{H}), 8.50-8.53$ (m, 1H, Ar-H); ${ }^{13} \mathrm{C}\left\{{ }^{1} \mathrm{H}\right\} \mathrm{NMR}(100 \mathrm{MHz}$, $\left.\mathrm{CDCl}_{3}\right) \delta 125.5,126.8,127.8,128.3,129.2,130.0,130.6$, $131.5,131.6,136.8,138.0,154.1,182.7$; distortionless enhancement by polarization transfer (DEPT)-135 NMR $\left(100 \mathrm{MHz}, \mathrm{CDCl}_{3}\right) \delta 125.5(\mathrm{CH}), 126.8(2 \times \mathrm{CH}), 127.8$ $(\mathrm{CH}), 128.3(\mathrm{CH}), 129.2(2 \times \mathrm{CH}), 130.0(\mathrm{CH}), 130.6$ $(\mathrm{CH}), 131.5(\mathrm{CH}) ;{ }^{77} \mathrm{Se} \mathrm{NMR}\left(76 \mathrm{MHz}, \mathrm{CDCl}_{3}\right) \delta 391.4$ (d, J 10.3 Hz); MS (relative intensity / \%) m/z, 102 (20.3), 156 (56.7), 184 (100.0), 258 (29.9), 286 (85.8); HRMS (APCI-QTOF) $\mathrm{m} / \mathrm{z}$, calcd. for $\mathrm{C}_{15} \mathrm{H}_{11} \mathrm{OSe}[\mathrm{M}+\mathrm{H}]^{+}$: 286.9975, found: 286.9978 .
2-(4-Tolyl)-4H-selenochromen-4-one $(\mathbf{3 b})^{6}$

Yield: $0.068 \mathrm{~g} \mathrm{(90 \% );} \mathrm{yellowish} \mathrm{solid;} \mathrm{mp} 136-138^{\circ} \mathrm{C}$; ${ }^{1} \mathrm{H}$ NMR (400 MHz, $\mathrm{CDCl}_{3}$ ) $\delta 2.39$ (s, 3H, Ar-CH $), 7.25$ (d, J 7.8 Hz, 2H, Ar-H), 7.34 (s, 1H, Ar-H), 7.46-7.53 (m, 4H, Ar-H), 7.62-7.67 (m, 1H, Ar-H), 8.57-8.61 (m, 1H, Ar$\mathrm{H}) ;{ }^{13} \mathrm{C}\left\{{ }^{1} \mathrm{H}\right\} \mathrm{NMR}\left(100 \mathrm{MHz}, \mathrm{CDCl}_{3}\right) \delta 21.2,124.9,126.6$, 127.6, 128.2, 129.9, 131.4, 131.7, 135.1, 136.8, 141.1, 154.0, 182.7; DEPT-135 NMR (100 MHz, $\left.\mathrm{CDCl}_{3}\right) \delta 21.2$ $\left(\mathrm{CH}_{3}\right), 124.9(\mathrm{CH}), 126.6(2 \times \mathrm{CH}), 127.6(\mathrm{CH}), 128.2$ (CH), $129.9(3 \times \mathrm{CH}), 131.4(\mathrm{CH}) ;{ }^{77} \mathrm{Se} \mathrm{NMR}(76 \mathrm{MHz}$, $\left.\mathrm{CDCl}_{3}\right) \delta 388.3(\mathrm{~d}, J 10.8 \mathrm{~Hz})$; MS (relative intensity / \%) m/z, 115 (54.9), 156 (48.1), 184 (100.0), 272 (13.5), 300 (58.8); HRMS (APCI-QTOF) $m / z$, calcd. for $\mathrm{C}_{16} \mathrm{H}_{13} \mathrm{OSe}$ $[\mathrm{M}+\mathrm{H}]^{+}:$301.0132, found: 301.0127 .

\section{2-(2-Tolyl)-4H-selenochromen-4-one (3c)}

Yield: $0.074 \mathrm{~g}$ (98\%); yellow oil; ${ }^{1} \mathrm{H}$ NMR (400 MHz, $\left.\mathrm{CDCl}_{3}\right) \delta 2.38\left(\mathrm{~s}, 3 \mathrm{H}, \mathrm{Ar}-\mathrm{CH}_{3}\right), 7.05$ (s, 1H, Ar-H), 7.24-7.35 (m, 4H, Ar-H), 7.49-7.56 (m, 2H, Ar-H), 7.64-7.66 (m, 1H, Ar-H), 8.62-8.67 (m, 1H, Ar-H); ${ }^{13} \mathrm{C}\left\{{ }^{1} \mathrm{H}\right\}$ NMR $\left(100 \mathrm{MHz}, \mathrm{CDCl}_{3}\right) \delta 19.9,125.9,127.7$, $128.1,128.3,128.5,129.5,130.1,130.8,131.5,131.7$, 135.0, 137.5, 137.7, 154.5, 182.3; DEPT-135 NMR $\left(100 \mathrm{MHz}, \mathrm{CDCl}_{3}\right) \delta 19.9\left(\mathrm{CH}_{3}\right), 125.9(\mathrm{CH}), 127.7(\mathrm{CH})$, $128.1(\mathrm{CH}), 128.3(\mathrm{CH}), 128.5(\mathrm{CH}), 129.5(\mathrm{CH}), 130.1$ (CH), $130.8(\mathrm{CH}), 131.5(\mathrm{CH}) ;{ }^{77} \mathrm{Se} \mathrm{NMR}\left(76 \mathrm{MHz}, \mathrm{CDCl}_{3}\right)$ $\delta 414.5(\mathrm{~d}, J 9.8 \mathrm{~Hz})$; MS (relative intensity / \%) $\mathrm{m} / \mathrm{z}, 115$ (41.9), 156 (47.3), 184 (100.0), 219 (73.3), 271 (7.6), 300 (90.8); HRMS (APCI-QTOF) $m / z$, calcd. for $\mathrm{C}_{16} \mathrm{H}_{13} \mathrm{OSe}$ $[\mathrm{M}+\mathrm{H}]^{+}:$301.0132, found: 301.0131 .

\section{2-(4-Chlorophenyl)-4H-selenochromen-4-one (3d) ${ }^{6}$}

Yield: $0.049 \mathrm{~g} \mathrm{(61 \% );} \mathrm{yellowish} \mathrm{solid;} \mathrm{mp} 135-137^{\circ} \mathrm{C}$; ${ }^{1} \mathrm{H} \mathrm{NMR}\left(400 \mathrm{MHz}, \mathrm{CDCl}_{3}\right) \delta 7.31$ (s, 1H, Ar-H), 7.45 (d, $J$ 8.6 Hz, 2H, Ar-H), 7.49-7.56 (m, 4H, Ar-H), 7.66-7.68 $(\mathrm{m}, 1 \mathrm{H}, \mathrm{Ar}-\mathrm{H}), 8.58-8.61(\mathrm{~m}, 1 \mathrm{H}, \mathrm{Ar}-\mathrm{H}) ;{ }^{13} \mathrm{C}\left\{{ }^{1} \mathrm{H}\right\} \mathrm{NMR}$ $\left(100 \mathrm{MHz}, \mathrm{CDCl}_{3}\right) \delta 125.8,127.9,128.1,128.2,129.5$, 130.1, 131.6, 131.7, 136.47, 136.49, 136.9, 152.3, 182.6; DEPT-135 NMR (100 MHz, $\left.\mathrm{CDCl}_{3}\right) \delta 125.8(\mathrm{CH}), 127.9$ (CH), $128.1(2 \times \mathrm{CH}), 128.2(\mathrm{CH}), 129.5(2 \times \mathrm{CH}), 130.1$ (CH), $131.7(\mathrm{CH}) ;{ }^{77} \mathrm{Se} \mathrm{NMR}\left(76 \mathrm{MHz}, \mathrm{CDCl}_{3}\right) \delta 389.6$ (d, $J 11.2 \mathrm{~Hz}$ ); MS (relative intensity / \%) $\mathrm{m} / \mathrm{z}, 136$ (6.9), 156 (42.3), 184 (100.0), 292 (21.1), 320 (76.4); HRMS (APCI-QTOF) $\mathrm{m} / z$, calcd. for $\mathrm{C}_{15} \mathrm{H}_{10} \mathrm{ClOSe}[\mathrm{M}+\mathrm{H}]^{+}$: 320.9585 , found: 320.9579 .

\section{2-(2-Chlorophenyl)-4H-selenochromen-4-one (3e)}

Yield: $0.061 \mathrm{~g}(76 \%)$; orange solid; $\mathrm{mp} 118-119{ }^{\circ} \mathrm{C}$; ${ }^{1} \mathrm{H} \mathrm{NMR}\left(400 \mathrm{MHz}, \mathrm{CDCl}_{3}\right) \delta 7.15$ (s, 1H, Ar-H), 7.31-7.43 (m, 3H, Ar-H), 7.48-7.58 (m, 3H, Ar-H), 7.63-7.69 (m, 1H, Ar-H), 8.62-8.65 (m, 1H, Ar-H); ${ }^{13} \mathrm{C}\left\{{ }^{1} \mathrm{H}\right\}$ NMR (100 MHz, 
$\left.\mathrm{CDCl}_{3}\right) \delta 127.0,127.8,128.1,129.3,130.1,130.3,130.4$ $130.8,131.57,131.6,132.0,136.6,137.5,151.0,182.3$; DEPT-135 NMR (100 MHz, $\left.\mathrm{CDCl}_{3}\right) \delta 127.0(\mathrm{CH}), 127.8$ $(\mathrm{CH}), 128.1(\mathrm{CH}), 129.3(\mathrm{CH}), 130.1(\mathrm{CH}), 130.3(\mathrm{CH})$, $130.4(\mathrm{CH}), 130.8(\mathrm{CH}), 131.6(\mathrm{CH}) ;{ }^{77} \mathrm{Se} \mathrm{NMR}(76 \mathrm{MHz}$, $\left.\mathrm{CDCl}_{3}\right) \delta 419.2(\mathrm{~d}, J 10.2 \mathrm{~Hz})$; MS (relative intensity / \%) m/z, 136 (5.2), 156 (41.3), 184 (100.0), 292 (15.3), 320 (76.9); HRMS (APCI-QTOF) $m / z$, calcd. for $\mathrm{C}_{15} \mathrm{H}_{10} \mathrm{ClOSe}$ $[\mathrm{M}+\mathrm{H}]^{+}:$320.9585, found: 320.9583 .

\section{2-(Naphthalen-2-yl)-4H-selenochromen-4-one (3f)}

Yield: $0.082 \mathrm{~g} \mathrm{(98 \% );} \mathrm{yellowish} \mathrm{solid;} \mathrm{mp} 132-133{ }^{\circ} \mathrm{C}$; ${ }^{1} \mathrm{H}$ NMR (400 MHz, $\mathrm{CDCl}_{3}$ ) $\delta 7.29$ (s, 1H, Ar-H), 7.46-7.54 (m, 6H, Ar-H), 7.60-7.65 (m, 1H, Ar-H), 7.87-7.91 (m, 2H, Ar-H), 8.08-8.10 (m, 1H, Ar-H), 8.67-8.71 (m, 1H, $\mathrm{Ar}-\mathrm{H}) ;{ }^{13} \mathrm{C}\left\{{ }^{1} \mathrm{H}\right\} \mathrm{NMR}\left(100 \mathrm{MHz}, \mathrm{CDCl}_{3}\right) \delta 124.9,125.0$, 126.49, 126.5, 127.0, 127.8, 128.0, 128.4, 129.4, 130.05, 130.08, 130.1, 131.5, 131.8, 133.6, 135.4, 137.9, 153.1, 182.2; DEPT-135 NMR (100 MHz, $\left.\mathrm{CDCl}_{3}\right) \delta 124.9(\mathrm{CH})$, $125.0(\mathrm{CH}), 126.49(\mathrm{CH}), 126.5(\mathrm{CH}), 127.0(\mathrm{CH}), 127.8$ $(\mathrm{CH}), 128.0(\mathrm{CH}), 128.4(\mathrm{CH}), 129.4(\mathrm{CH}), 130.08(\mathrm{CH})$, $130.11(\mathrm{CH}), 131.5(\mathrm{CH}) ;{ }^{77} \mathrm{Se} \mathrm{NMR}\left(76 \mathrm{MHz}, \mathrm{CDCl}_{3}\right)$ $\delta 427.3$ (d, J 9.2 Hz); MS (relative intensity / \%) $\mathrm{m} / \mathrm{z}, 152$ (96.0), 156 (30.7), 184 (34.8), 319 (100.0), 336 (35.5); HRMS (APCI-QTOF) $m / z$, calcd. for $\mathrm{C}_{19} \mathrm{H}_{13} \mathrm{OSe}[\mathrm{M}+\mathrm{H}]^{+}$: 337.0132, found: 337.0125.

\section{(E)-2-Styryl-4H-selenochromen-4-one (3g)}

Yield: $0.058 \mathrm{~g}$ (74\%); yellowish solid; $\mathrm{mp} 133-135^{\circ} \mathrm{C}$; ${ }^{1} \mathrm{H}$ NMR (400 MHz, $\left.\mathrm{CDCl}_{3}\right) \delta 7.08(\mathrm{~d}, J 16.1 \mathrm{~Hz}, 1 \mathrm{H}$, $\mathrm{CH})$, 7.14-7.18 (m, 2H, Ar-H), 7.34-7.42 (m, 3H, Ar-H), 7.45-7.54 (m, 4H, Ar-H), 7.63-7.65 (m, 1H, Ar-H), 8.54 (dd, J 7.9 and $1.4 \mathrm{~Hz}, 1 \mathrm{H}, \mathrm{Ar}-\mathrm{H}) ;{ }^{13} \mathrm{C}\left\{{ }^{1} \mathrm{H}\right\} \mathrm{NMR}(100 \mathrm{MHz}$, $\left.\mathrm{CDCl}_{3}\right) \delta 127.0,127.4,127.6,127.7,128.3,129.0,129.6$, $129.9,131.7,132.2,135.1,136.1,149.6,183.0$; DEPT$135 \mathrm{NMR}\left(100 \mathrm{MHz}, \mathrm{CDCl}_{3}\right) \delta 127.0(\mathrm{CH}), 127.4(2 \times \mathrm{CH})$, $127.6(\mathrm{CH}), 127.7(\mathrm{CH}), 128.3(\mathrm{CH}), 129.0(2 \times \mathrm{CH}), 129.6$ $(\mathrm{CH}), 129.9(\mathrm{CH}), 131.7(\mathrm{CH}), 136.1(\mathrm{CH}) ;{ }^{77} \mathrm{Se} \mathrm{NMR}$ $\left(76 \mathrm{MHz}, \mathrm{CDCl}_{3}\right) \delta 360.2(\mathrm{t}, J 9.8 \mathrm{~Hz}$ ); MS (relative intensity / \%) $\mathrm{m} / \mathrm{z}, 128$ (60.6), 156 (39.5), 184 (100.0), 295 (9.3), 312 (37.0); HRMS (APCI-QTOF) $\mathrm{m} / z$, calcd. for $\mathrm{C}_{17} \mathrm{H}_{13} \mathrm{OSe}[\mathrm{M}+\mathrm{H}]^{+}: 313.0132$, found: 313.0122 .

\section{2-Pentyl-4H-selenochromen-4-one (3h) ${ }^{6}$}

Yield: $0.068 \mathrm{~g}$ (97\%); yellowish solid; $\mathrm{mp} 38-39{ }^{\circ} \mathrm{C}$; ${ }^{1} \mathrm{H} \mathrm{NMR}\left(400 \mathrm{MHz}, \mathrm{CDCl}_{3}\right) \delta 0.90\left(\mathrm{t}, J 7.4 \mathrm{~Hz}, 3 \mathrm{H}, \mathrm{CH}_{3}\right)$, 1.31-1.41 (m, 4H, $\mathrm{CH}_{2}$ ), 1.72 (quint, J 7.4 Hz, 2H, $\mathrm{CH}_{2}$ ), 2.73 (t, J 7.4 Hz, 2H, $\mathrm{CH}_{2}$ ), 7.01 (s, 1H, Ar-H), 7.44-7.51 (m, 2H, Ar-H), 7.58-7.63 (m, 1H, Ar-H), 8.55-8.57 (m, 1H, $\mathrm{Ar}-\mathrm{H}) ;{ }^{13} \mathrm{C}\left\{{ }^{1} \mathrm{H}\right\} \mathrm{NMR}\left(100 \mathrm{MHz}, \mathrm{CDCl}_{3}\right) \delta 13.8,22.2,29.7$, 30.9, 38.9, 126.0, 127.4, 128.3, 129.9, 131.2, 131.8, 136.5,
158.2, 182.5; DEPT-135 NMR (100 MHz, $\left.\mathrm{CDCl}_{3}\right) \delta 13.8$ $\left(\mathrm{CH}_{3}\right), 22.2\left(\mathrm{CH}_{2}\right), 29.7\left(\mathrm{CH}_{2}\right), 30.9\left(\mathrm{CH}_{2}\right), 38.9\left(\mathrm{CH}_{2}\right)$, $126.0(\mathrm{CH}), 127.4(\mathrm{CH}), 128.3(\mathrm{CH}), 129.9(\mathrm{CH}), 131.2$ $(\mathrm{CH}) ;{ }^{77} \mathrm{Se} \mathrm{NMR}\left(76 \mathrm{MHz}, \mathrm{CDCl}_{3}\right) \delta 400.5-401.0(\mathrm{~m}) ; \mathrm{MS}$ (relative intensity /\%) m/z, 95 (5.0), 115 (43.9), 156 (35.2), 184 (100.0), 224 (79.0), 280 (34.0); HRMS (APCI-QTOF) $m / z$, calcd. for $\mathrm{C}_{14} \mathrm{H}_{17} \mathrm{OSe}[\mathrm{M}+\mathrm{H}]^{+}: 281.0445$, found: 281.0438 .

\section{7-Chloro-2-phenyl-4H-selenochromen-4-one (3i)}

Yield: $0.058 \mathrm{~g}(72 \%)$; yellowish solid; $\mathrm{mp} 148-149^{\circ} \mathrm{C}$; ${ }^{1} \mathrm{H}$ NMR (400 MHz, $\mathrm{CDCl}_{3}$ ) $\delta 7.33$ (s, 1H, Ar-H), 7.44-7.51 (m, 4H, Ar-H), 7.59-7.61 (m, 2H, Ar-H), 7.67 (d, J 2.0 Hz, $1 \mathrm{H}, \mathrm{Ar}-\mathrm{H}), 8.51$ (d, J $8.7 \mathrm{~Hz}, 1 \mathrm{H}, \mathrm{Ar}-\mathrm{H}) ;{ }^{13} \mathrm{C}\left\{{ }^{1} \mathrm{H}\right\} \mathrm{NMR}$ $\left(100 \mathrm{MHz}, \mathrm{CDCl}_{3}\right) \delta 125.6,126.8,127.6,128.4,129.3$, 130.0, 130.9, 131.4, 137.7, 137.9, 138.3, 153.7, 181.8; DEPT-135 NMR (100 MHz, $\left.\mathrm{CDCl}_{3}\right) \delta 125.6(\mathrm{CH}), 126.8$ $(2 \times \mathrm{CH}), 127.6(\mathrm{CH}), 128.4(\mathrm{CH}), 129.3(2 \times \mathrm{CH}), 130.9$ $(\mathrm{CH}), 131.4(\mathrm{CH}) ;{ }^{77} \mathrm{Se} \mathrm{NMR}\left(76 \mathrm{MHz}, \mathrm{CDCl}_{3}\right) \delta 396.5$ (d, $J 8.5 \mathrm{~Hz}$ ); MS (relative intensity / \%) $\mathrm{m} / \mathrm{z}, 102$ (14.4), 190 (32.6), 218 (100.0), 292 (29.1), 320 (84.5); HRMS (APCI-QTOF) $m / z$, calcd. for $\mathrm{C}_{15} \mathrm{H}_{10} \mathrm{ClOSe}[\mathrm{M}+\mathrm{H}]^{+}$: 320.9585 , found: 320.9578 .

\section{2-Phenyl-4H-thiochromen-4-one (4a) ${ }^{6}$}

Yield: $0.059 \mathrm{~g}$ (99\%); yellowish solid; mp $95-96{ }^{\circ} \mathrm{C}$; ${ }^{1} \mathrm{H} \mathrm{NMR}\left(400 \mathrm{MHz}, \mathrm{CDCl}_{3}\right.$ ) $\delta 7.24$ (s, 1H, Ar-H), 7.47-7.56 (m, 4H, Ar-H), 7.59-7.70 (m, 4H, Ar-H), 8.54 (d, J 7.8 Hz, $1 \mathrm{H}, \mathrm{Ar}-\mathrm{H}) ;{ }^{13} \mathrm{C}\left\{{ }^{1} \mathrm{H}\right\} \mathrm{NMR}\left(100 \mathrm{MHz}, \mathrm{CDCl}_{3}\right) \delta 123.4$, 126.4, 126.9, 127.7, 128.5, 129.2, 130.76, 130.8, 131.5, 136.5, 137.6, 153.0, 180.8; DEPT-135 NMR (100 MHz, $\left.\mathrm{CDCl}_{3}\right) \delta 123.4(\mathrm{CH}), 126.4(\mathrm{CH}), 126.9(2 \times \mathrm{CH}), 127.7$ $(\mathrm{CH}), 128.5(\mathrm{CH}), 129.2(2 \times \mathrm{CH}), 130.76(\mathrm{CH}), 131.5$ (CH); MS (relative intensity / \%) $\mathrm{m} / \mathrm{z}, 102$ (6.4), 108 (53.9), 136 (57.5), 210 (94.5), 238 (100.0); HRMS (APCI-QTOF) $m / z$, calcd. for $\mathrm{C}_{15} \mathrm{H}_{11} \mathrm{OS}[\mathrm{M}+\mathrm{H}]^{+}: 239.0531$, found: 239.0523 .

\section{2-(4-Tolyl)-4H-thiochromen-4-one (4b) ${ }^{6}$}

Yield: $0.047 \mathrm{~g}(75 \%)$; gray solid; $\mathrm{mp} 109-110{ }^{\circ} \mathrm{C}$; ${ }^{1} \mathrm{H}$ NMR $\left(400 \mathrm{MHz}, \mathrm{CDCl}_{3}\right) \delta 2.42\left(\mathrm{~s}, 3 \mathrm{H}, \mathrm{Ar}-\mathrm{CH}_{3}\right), 7.23$ (s, 1H, Ar-H), 7.30 (d, J 8.0 Hz, 2H, Ar-H), 7.52-7.66 (m, $5 \mathrm{H}, \mathrm{Ar}-\mathrm{H}), 8.54$ (d, J 7.9 Hz, $1 \mathrm{H}, \mathrm{Ar}-\mathrm{H}) ;{ }^{13} \mathrm{C}\left\{{ }^{1} \mathrm{H}\right\} \mathrm{NMR}$ $\left(100 \mathrm{MHz}, \mathrm{CDCl}_{3}\right) \delta 21.3,122.8,126.4,126.8,127.6$, $128.5,129.9,130.9,131.5,133.7,137.7,141.3,153.1$, 180.9; DEPT-135 NMR (100 MHz, $\mathrm{CDCl}_{3}$ ) $\delta 21.3$ $\left(\mathrm{CH}_{3}\right), 122.8(\mathrm{CH}), 126.4(\mathrm{CH}), 126.8(2 \times \mathrm{CH}), 127.6$ $(\mathrm{CH}), 128.5(\mathrm{CH}), 129.9(2 \times \mathrm{CH}), 131.5(\mathrm{CH}) ; \mathrm{MS}$ (relative intensity / \%) m/z, 108 (51.7), 115 (30.8), 136 (65.5), 224 (91.8), 252 (100.0); HRMS (APCI-QTOF) $\mathrm{m} / \mathrm{z}$, calcd. for $\mathrm{C}_{16} \mathrm{H}_{13} \mathrm{OS}[\mathrm{M}+\mathrm{H}]^{+}: 253.0687$, found: 253.0677 . 
2-(2-Tolyl)-4H-thiochromen-4-one (4c) ${ }^{6}$

Yield: $0.051 \mathrm{~g}(81 \%)$; yellow oil; ${ }^{1} \mathrm{H}$ NMR $(400 \mathrm{MHz}$, $\left.\mathrm{CDCl}_{3}\right) \delta 2.38\left(\mathrm{~s}, 3 \mathrm{H}, \mathrm{Ar}-\mathrm{CH}_{3}\right), 6.92$ (s, 1H, Ar-H), 7.25-7.38 (m, 4H, Ar-H), 7.53-7.57 (m, 1H, Ar-H), 7.59-7.64 (m, 2H, Ar-H), $8.58(\mathrm{dt}, J 8.0$ and $1.0 \mathrm{~Hz}, 1 \mathrm{H}$, Ar-H); ${ }^{13} \mathrm{C}\left\{{ }^{1} \mathrm{H}\right\}$ NMR (100 MHz, $\left.\mathrm{CDCl}_{3}\right) \delta 19.8,126.0$, 126.1, 126.2, 127.7, 128.6, 128.9, 129.7, 130.8, 130.84, $131.5,135.6,136.0,138.3,153.5,180.4$; DEPT-135 NMR $\left(100 \mathrm{MHz}, \mathrm{CDCl}_{3}\right) \delta 19.8\left(\mathrm{CH}_{3}\right), 126.0(\mathrm{CH}), 126.1(\mathrm{CH})$, $126.2(\mathrm{CH}), 127.7(\mathrm{CH}), 128.6(\mathrm{CH}), 128.9(\mathrm{CH}), 129.7$ $(\mathrm{CH}), 130.8(\mathrm{CH}), 131.5(\mathrm{CH})$; MS (relative intensity / \%) m/z, 108 (34.7), 115 (29.5), 136 (45.9), 223 (38.4), 252 (100.0); HRMS (APCI-QTOF) $m / z$, calcd. for $\mathrm{C}_{16} \mathrm{H}_{13} \mathrm{OS}$ $[\mathrm{M}+\mathrm{H}]^{+}:$253.0687, found: 253.0685 .

\section{2-(4-Chlorophenyl)-4H-thiochromen-4-one (4d) ${ }^{6}$}

Yield: $0.042 \mathrm{~g}(62 \%)$; yellowish solid; $\mathrm{mp} 159-160{ }^{\circ} \mathrm{C}$; ${ }^{1} \mathrm{H}$ NMR (400 MHz, $\mathrm{CDCl}_{3}$ ) $\delta 7.18(\mathrm{~s}, 1 \mathrm{H}, \mathrm{Ar}-\mathrm{H}), 7.46$ (d, $J$ 8.5 Hz, 2H, Ar-H), 7.52-7.56 (m, 1H, Ar-H), 7.60-7.65 (m, 4H, Ar-H), 8.52 (d, J 7.7 Hz, 1H, Ar-H); ${ }^{13} \mathrm{C}\left\{{ }^{1} \mathrm{H}\right\}$ NMR $\left(100 \mathrm{MHz}, \mathrm{CDCl}_{3}\right) \delta 123.4,126.4,127.9,128.1,128.5$, $129.5,130.7,131.7,134.9,137.0,137.3,151.5,180.6$; DEPT-135 NMR (100 MHz, $\left.\mathrm{CDCl}_{3}\right) \delta 123.4(\mathrm{CH}), 126.4$ $(\mathrm{CH}), 127.9(\mathrm{CH}), 128.1(2 \times \mathrm{CH}), 128.5(\mathrm{CH}), 129.5$ (2× CH), $131.7(\mathrm{CH})$; MS (relative intensity / \%) $\mathrm{m} / \mathrm{z}$, 108 (46.3), 136 (80.3), 244 (90.3), 272 (100.0); HRMS (APCI-QTOF) $m / z$, calcd. for $\mathrm{C}_{15} \mathrm{H}_{10} \mathrm{ClOS}[\mathrm{M}+\mathrm{H}]^{+}$: 273.0141, found: 273.0150 .

\section{2-(2-Chlorophenyl)-4H-thiochromen-4-one (4e) ${ }^{6}$}

Yield: $0.057 \mathrm{~g}(83 \%)$; white solid; $\mathrm{mp} 126-127{ }^{\circ} \mathrm{C}$; ${ }^{1} \mathrm{H}$ NMR $\left(400 \mathrm{MHz}, \mathrm{CDCl}_{3}\right) \delta 7.02$ (s, 1H, Ar-H), 7.36-7.46 (m, 3H, Ar-H), 7.51-7.59 (m, 2H, Ar-H), 7.62-7.67 (m, $2 \mathrm{H}, \mathrm{Ar}-\mathrm{H}), 8.58$ (d, J $8.0 \mathrm{~Hz}, 1 \mathrm{H}, \mathrm{Ar}-\mathrm{H}) ;{ }^{13} \mathrm{C}\left\{{ }^{1} \mathrm{H}\right\} \mathrm{NMR}$ $\left(100 \mathrm{MHz}, \mathrm{CDCl}_{3}\right) \delta 126.3,127.1,127.2,127.8,128.7$, $130.5,130.7,130.9,131.1,131.7,132.6,135.2,138.1$, 150.4, 180.4; DEPT-135 NMR (100 MHz, $\left.\mathrm{CDCl}_{3}\right) \delta 126.3$ $(\mathrm{CH}), 127.1(\mathrm{CH}), 127.2(\mathrm{CH}), 127.8(\mathrm{CH}), 128.7(\mathrm{CH})$, $130.5(\mathrm{CH}), 130.7(\mathrm{CH}), 131.1(\mathrm{CH}), 131.7(\mathrm{CH})$; MS (relative intensity / \%) m/z, 108 (41.9), 136 (73.0), 244 (71.4), 272 (100.0); HRMS (APCI-QTOF) $\mathrm{m} / \mathrm{z}$, calcd. for $\mathrm{C}_{15} \mathrm{H}_{10} \mathrm{ClOS}[\mathrm{M}+\mathrm{H}]^{+}:$273.0141, found: 273.0133.

\section{2-(Naphthalen-2-yl)-4H-thiochromen-4-one (4f) ${ }^{21}$}

Yield: $0.068 \mathrm{~g}(94 \%)$; yellowish solid; $\mathrm{mp} 135-136^{\circ} \mathrm{C}$; ${ }^{1} \mathrm{H}$ NMR $\left(400 \mathrm{MHz}, \mathrm{CDCl}_{3}\right) \delta 7.15$ (s, 1H, Ar-H), 7.47-7.63 (m, 7H, Ar-H), 7.88-7.95 (m, 2H, Ar-H), 8.04-8.06 (m, 1H, Ar-H), 8.62 (d, J 7.7 Hz, 1H); ${ }^{13} \mathrm{C}\left\{{ }^{1} \mathrm{H}\right\}$ NMR (100 MHz, $\left.\mathrm{CDCl}_{3}\right) \delta 124.8,124.9,126.1,126.5,127.06,127.12,127.2$, $127.7,128.4,128.6,130.3,130.4,131.0,131.5,133.6$, 133.9, 138.5, 152.2, 180.2; DEPT-135 NMR (100 MHz,
$\left.\mathrm{CDCl}_{3}\right) \delta 124.8(\mathrm{CH}), 124.9(\mathrm{CH}), 126.1(\mathrm{CH}), 126.5(\mathrm{CH})$, $127.06(\mathrm{CH}), 127.12(\mathrm{CH}), 127.2(\mathrm{CH}), 127.7(\mathrm{CH}), 128.4$ (CH), $128.6(\mathrm{CH}), 130.3(\mathrm{CH}), 131.5(\mathrm{CH})$; MS (relative intensity / \%) m/z, 108 (9.5), 136 (6.3), 152 (21.1), 271 (100.0), 288 (23.3); HRMS (APCI-QTOF) $\mathrm{m} / \mathrm{z}$, calcd. for $\mathrm{C}_{19} \mathrm{H}_{13} \mathrm{OS}[\mathrm{M}+\mathrm{H}]^{+}: 289.0687$, found: 289.0680 .

\section{(E)-2-Styryl-4H-thiochromen-4-one (4g) ${ }^{6}$}

Yield: $0.040 \mathrm{~g}(61 \%)$; yellowish solid; $\mathrm{mp} 119-120{ }^{\circ} \mathrm{C}$; ${ }^{1} \mathrm{H}$ NMR (400 MHz, $\mathrm{CDCl}_{3}$ ) $\delta 7.0(\mathrm{~s}, 1 \mathrm{H}, \mathrm{Ar}-\mathrm{H}), 7.04$ (d, $J 16.2 \mathrm{~Hz}, 1 \mathrm{H}, \mathrm{CH}), 7.31$ (d, J 16.2 Hz, 1H, CH), 7.35-7.44 (m, 3H, Ar-H), 7.49-7.64 (m, 5H, Ar-H), 8.48-8.50 (m, 1H, Ar-H); ${ }^{13} \mathrm{C}\left\{{ }^{1} \mathrm{H}\right\}$ NMR $\left(100 \mathrm{MHz}, \mathrm{CDCl}_{3}\right) \delta 124.6,125.9$, 126.4, 127.4, 127.5, 128.5, 129.0, 129.7, 131.3, 131.7, 135.1, 135.6, 136.6, 149.2, 181.1; DEPT-135 NMR (100 MHz, $\left.\mathrm{CDCl}_{3}\right) \delta 124.6(\mathrm{CH}), 125.9(\mathrm{CH}), 126.4(\mathrm{CH}), 127.4$ $(2 \times \mathrm{CH}), 127.5(\mathrm{CH}), 128.5(\mathrm{CH}), 129.0(2 \times \mathrm{CH}), 129.7$ (CH), $131.7(\mathrm{CH}), 135.6(\mathrm{CH})$; MS (relative intensity / \%) m/z, 108 (52.8), 128 (40.6), 136 (39.8), 247 (59.9), 263 (100.0), 264 (72.1); HRMS (APCI-QTOF) $m / z$, calcd. for $\mathrm{C}_{17} \mathrm{H}_{13} \mathrm{OS}[\mathrm{M}+\mathrm{H}]^{+}: 265.0687$, found: 265.0683 .

\section{2-Pentyl-4H-thiochromen-4-one (4h) ${ }^{7}$}

Yield: $0.053 \mathrm{~g}(91 \%)$; yellowish solid; $\mathrm{mp} 36-37{ }^{\circ} \mathrm{C}$; ${ }^{1} \mathrm{H}$ NMR (400 MHz, $\left.\mathrm{CDCl}_{3}\right) \delta 0.91\left(\mathrm{t}, J 7.3 \mathrm{~Hz}, 3 \mathrm{H}, \mathrm{CH}_{3}\right.$ ), 1.35-1.39 (m, 4H, $\mathrm{CH}_{2}$ ), 1.73 (quint, J $7.3 \mathrm{~Hz}, 2 \mathrm{H}, \mathrm{CH}_{2}$ ), 2.67 (t, J 7.3 Hz, 2H, $\left.\mathrm{CH}_{2}\right), 6.86$ (s, 1H, Ar-H), 7.47-7.52 (m, 1H, Ar-H), 7.55-7.59 (m, 2H, Ar-H), 8.50 (d, J 8.0 Hz, $1 \mathrm{H}, \mathrm{Ar}-\mathrm{H}) ;{ }^{13} \mathrm{C}\left\{{ }^{1} \mathrm{H}\right\} \mathrm{NMR}\left(100 \mathrm{MHz}, \mathrm{CDCl}_{3}\right) \delta 13.8$, 22.2, 29.4, 30.9, 37.3, 124.0, 126.1, 127.4, 128.4, 130.9, 131.2, 137.7, 156.5, 180.6; DEPT-135 NMR (100 MHz, $\left.\mathrm{CDCl}_{3}\right) \delta 13.8\left(\mathrm{CH}_{3}\right), 22.2\left(\mathrm{CH}_{2}\right), 29.4\left(\mathrm{CH}_{2}\right), 30.9\left(\mathrm{CH}_{2}\right)$, $37.3\left(\mathrm{CH}_{2}\right), 124.0(\mathrm{CH}), 126.1(\mathrm{CH}), 127.4(\mathrm{CH}), 128.4$ (CH), $131.2(\mathrm{CH})$; MS (relative intensity / \%) m/z, 95 (1.8), 108 (21.3), 136 (67.2), 176 (100.0), 232 (28.0); HRMS (APCI-QTOF) $m / z$, calcd. for $\mathrm{C}_{14} \mathrm{H}_{17} \mathrm{OS}[\mathrm{M}+\mathrm{H}]^{+}$: 233.1000, found: 233.0996 .

\section{7-Chloro-2-phenyl-4H-thiochromen-4-one (4i) ${ }^{7}$}

Yield: $0.056 \mathrm{~g} \mathrm{(83 \% );} \mathrm{yellowish} \mathrm{solid;} \mathrm{mp} 134-135{ }^{\circ} \mathrm{C}$; ${ }^{1} \mathrm{H} \mathrm{NMR}\left(400 \mathrm{MHz}, \mathrm{CDCl}_{3}\right) \delta 7.18(\mathrm{~s}, 1 \mathrm{H}, \mathrm{Ar}-\mathrm{H}), 7.44-7.53$ (m, 4H, Ar-H), 7.60 (d, J 1.8 Hz, 1H, Ar-H), 7.64 (dd, $J 7.6$ and $1.8 \mathrm{~Hz}, 2 \mathrm{H}, \mathrm{Ar}-\mathrm{H}), 8.44$ (d, J 8.7 Hz, 1H, Ar-H); ${ }^{13} \mathrm{C}\left\{{ }^{1} \mathrm{H}\right\}$ NMR $\left(100 \mathrm{MHz}, \mathrm{CDCl}_{3}\right) \delta 123.4,125.6,126.8$, 128.3, 129.2, 129.3, 130.1, 130.9, 136.0, 138.2, 138.9, 152.6, 179.8; DEPT-135 NMR (100 MHz, $\left.\mathrm{CDCl}_{3}\right) \delta 123.4(\mathrm{CH})$, $125.6(\mathrm{CH}), 126.8(2 \times \mathrm{CH}), 128.3(\mathrm{CH}), 129.2(2 \times \mathrm{CH})$, $130.1(\mathrm{CH}), 130.9(\mathrm{CH})$; MS (relative intensity / \%) $\mathrm{m} / \mathrm{z}$, 102 (6.9), 142 (20.1), 170 (39.5), 244 (92.1), 272 (100.0); HRMS (APCI-QTOF) $m / z$, calcd. for $\mathrm{C}_{15} \mathrm{H}_{10} \mathrm{ClOS}[\mathrm{M}+\mathrm{H}]^{+}$: 273.0141, found: 273.0141 . 
Procedure for identify the nucleophilic species formed through ${ }^{77}$ Se NMR spectroscopy

In a $10.0 \mathrm{~mL}$ reaction vial containing $\mathrm{Se}(0.3 \mathrm{mmol})$ in PEG-400 $(2.0 \mathrm{~mL})$ under argon atmosphere was added $\mathrm{NaBH}_{4}(0.7 \mathrm{mmol})$, and the mixture was slowly heated to $50{ }^{\circ} \mathrm{C}$ being stirred for $30 \mathrm{~min}$. Then, $500 \mu \mathrm{L}$ of this solution was transferred to a $5 \mathrm{~mm}$ NMR tube, purged with argon gas and a capillary tube with a solution of the diphenyl diselenide in $\mathrm{CDCl}_{3}$ was used as the chemical shift reference ( $\delta 463 \mathrm{ppm})$, after the NMR tube was capped with a rubber septum and analyzed by ${ }^{77} \mathrm{Se} \mathrm{NMR}$. The spectra were recorded at $298 \mathrm{~K}$ and with 845 scans.

\section{Results and Discussion}

Based on the results of our recent studies using elemental chalcogens, ${ }^{19}$ we chose selenium powder 1a, $\mathrm{NaBH}_{4}$ and 1-(2-chlorophenyl)-3-phenylprop-2-yn1-one 2a as model substrates to identify the optimum conditions under argon (Ar) atmosphere and PEG-400 as the solvent (Table 1). For this test, a mixture of selenium $(0.25 \mathrm{mmol})$ and $\mathrm{NaBH}_{4}$ (2.3 equiv) in PEG-400 $(2.0 \mathrm{~mL})$ was stirred at $50{ }^{\circ} \mathrm{C}$, for the in situ generation of the nucleophilic selenium species. This formation was monitored by the color change of the reaction medium, changing from grey to colorless after $0.5 \mathrm{~h}$ of stirring. After that, the 2-chlorophenyl ethynyl ketone $2 \mathrm{a}(0.25 \mathrm{mmol})$ was added to the reaction vessel and the temperature was raised to $100{ }^{\circ} \mathrm{C}$. In these conditions, the corresponding 2-phenyl-4H-selenochromen-4-one 3a was obtained with $56 \%$ gas chromatography (GC) yield in a reaction time of $2 \mathrm{~h}$ (Table 1, entry 1 ). To our delight, increasing the amount of selenium powder to $0.3 \mathrm{mmol}$ provides an improvement in the reaction performance, and the target compound $\mathbf{3 a}$ could be achieved with $99 \%$ GC yield and an isolated yield of $98 \%$ (Table 1, entry 2). Reducing the reaction time to $1 \mathrm{~h}$ showed a negative effect to produce the desired Se-flavone 3a, and only $69 \%$ GC yield was observed (Table 1, entry 3). We also investigated the effect of the temperature on the reaction yield. However, decreasing the temperature results in lower yields (Table 1, entries 4-6). Subsequently, other solvents were investigated under similar reaction conditions. Polar solvents such as EtOH, glycerol and water provided product $\mathbf{3 a}$, but in lower yields compared to the use of PEG-400, furthermore, several by-products were observed in gas chromatography mass spectrometry (GC-MS, Table 1, entries 7-9 vs. entry 2). Additionally, aprotic solvents were inefficient in our study and the use of dimethyl sulfoxide (DMSO) or MeCN failed to afford the desired product (Table 1, entries 10-11). Thus, we established the best reaction conditions as being a two steps onepot procedure, which starts with the reaction of selenium $1 \mathbf{a}(0.3 \mathrm{mmol})$ and $\mathrm{NaBH}_{4}(0.7 \mathrm{mmol})$ in PEG-400

Table 1. Optimization of the synthesis of Se-flavone $3 \mathbf{a}^{\mathrm{a}}$

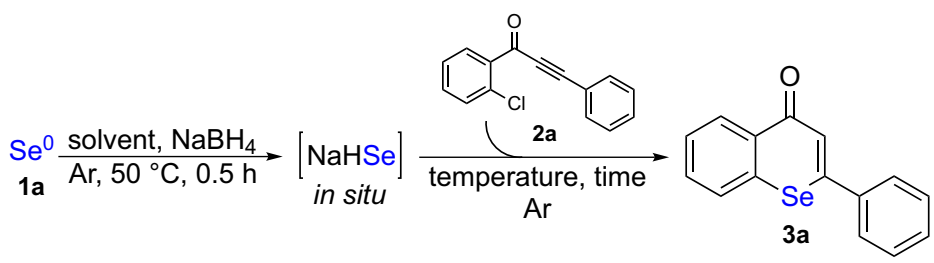

\begin{tabular}{|c|c|c|c|c|c|}
\hline entry & $\mathrm{Se}^{0} / \mathrm{mmol}$ & Solvent & Temperature $/{ }^{\circ} \mathrm{C}$ & time $/ \mathrm{h}$ & Yield $^{\mathrm{b}} / \%$ \\
\hline 1 & 0.25 & PEG-400 & 100 & 2 & 56 \\
\hline 2 & 0.30 & PEG-400 & 100 & 2 & $99(98)^{\mathrm{c}}$ \\
\hline 3 & 0.30 & PEG-400 & 100 & 1 & 69 \\
\hline 4 & 0.30 & PEG-400 & 80 & 2 & 82 \\
\hline 5 & 0.30 & PEG-400 & 50 & 2 & 76 \\
\hline 6 & 0.30 & PEG-400 & 25 & 2 & 57 \\
\hline 7 & 0.30 & $\mathrm{EtOH}$ & 78 & 2 & 32 \\
\hline 8 & 0.30 & glycerol & 100 & 2 & 12 \\
\hline 9 & 0.30 & $\mathrm{H}_{2} \mathrm{O}$ & 100 & 2 & 16 \\
\hline 10 & 0.30 & DMSO & 100 & 2 & NR \\
\hline 11 & 0.30 & $\mathrm{MeCN}$ & 82 & 2 & NR \\
\hline
\end{tabular}

a Reactions were performed using $\mathrm{Se}^{0} \mathbf{1 a}, \mathrm{NaBH}_{4}$ (2.33 equiv in relation to selenium powder) in $2.0 \mathrm{~mL}$ of the solvent at $50^{\circ} \mathrm{C}$ under argon for $0.5 \mathrm{~h}$. Then, $0.25 \mathrm{mmol}$ of $\mathbf{2 a}$ was added. ' $Y$ ield determined by GC using ${ }^{n}$ dodecane as an internal standard. ' $Y$ ield for the isolated product. PEG-400: polyethylene glycol-400; DMSO: dimethyl sulfoxide; NR: no reaction. 
(2.0 mL) at $50{ }^{\circ} \mathrm{C}$ under Ar for $0.5 \mathrm{~h}$. Then, compound 2a $(0.25 \mathrm{mmol})$ is added into the reaction medium, and the resulting mixture is stirred for an additional $2 \mathrm{~h}$ at $100{ }^{\circ} \mathrm{C}$ (Table 1, entry 2).

With the optimal reaction conditions identified, various 2-chloroaryl ethynyl ketone $\mathbf{2}$ for this ring closure reaction were investigated and the results are summarized in Scheme 2. In the beginning, the electronic effects on the substituent $\mathrm{R}^{1}$ of the compound $\mathbf{2}$ were investigated. Briefly, this method proved to be more efficient for electron-donating substituents when compared with electron-withdrawing substituents in the aromatic ring. When using electron-donating groups in the aromatic ring $\left(2-\mathrm{CH}_{3}\right.$ and $\left.4-\mathrm{CH}_{3}\right)$, the corresponding Se-flavones $\mathbf{3 b}$ and $3 \mathbf{c}$ were obtained in high yields. Since by using electronwithdrawing atoms in the aromatic ring $(2-\mathrm{Cl}$ and $4-\mathrm{Cl})$, the Se-flavones 3d and 3e were obtained 61 and 76\%, respectively. To our delight, the substitution of the aryl group by naphthyl group did not affect the efficiency of this transformation, and the Se-flavone $\mathbf{3 f}$ was obtained in an excellent yield of $98 \%$. In the same manner, substrate possessing styryl moiety can also be effectively converted into Se-flavone functionalized with styryl derivative $\mathbf{3 g}$ in $74 \%$ yield under well-established conditions. Despite employing a reduction system $\left(\mathrm{NaBH}_{4} / \mathrm{PEG}-400\right)$, this method proved to be chemoselective, since no reaction in the carbon-carbon double bond of the styryl group was observed. We can also prepare an analogous compound with an aliphatic chain, through the reaction compound $\mathbf{2 h}$, leading to the formation of the product $3 \mathbf{h}$ in an excellent yield of $97 \%$. We also explored the $\mathrm{R}$ position of the 2-chloro ethynyl ketone 2 , substrate $2 \mathbf{i}(\mathrm{R}=\mathrm{Cl})$ reacted under well-established conditions, affording the desired product $3 \mathbf{i}$ in $72 \%$ yield.

In addition, we explored the change of the chlorine atom by bromine atom in the substrate $\mathbf{2}$ under optimized conditions. After $2 \mathrm{~h}$ of reaction time, the Se-flavone 3a was also obtained in a good yield, however lower than the chlorine one. This result is consistent because it clearly supports the point about halogen's atom role, in which electron-withdrawing groups improve the reactivity for the aromatic nucleophilic substitution reaction. Considering the straightforward method herein developed, it was successfully applied in the gram-scale synthesis of Se-flavone 3a (4 mmol), leading to $81 \%$ yield of the corresponding product.

Encouraged by the Se-flavones results, we next studied the general applicability of the method for the synthesis of thioflavones 4 by the reaction of sulfur powder $\mathbf{1 b}$ with a variety of 2-chloroaryl ethynyl ketones $\mathbf{2}$, as shown in the Scheme 3. Thus, when using the compound 2a, with a neutral group, the 2-phenyl-4H-thiochromen-4-one $\mathbf{4 a}$
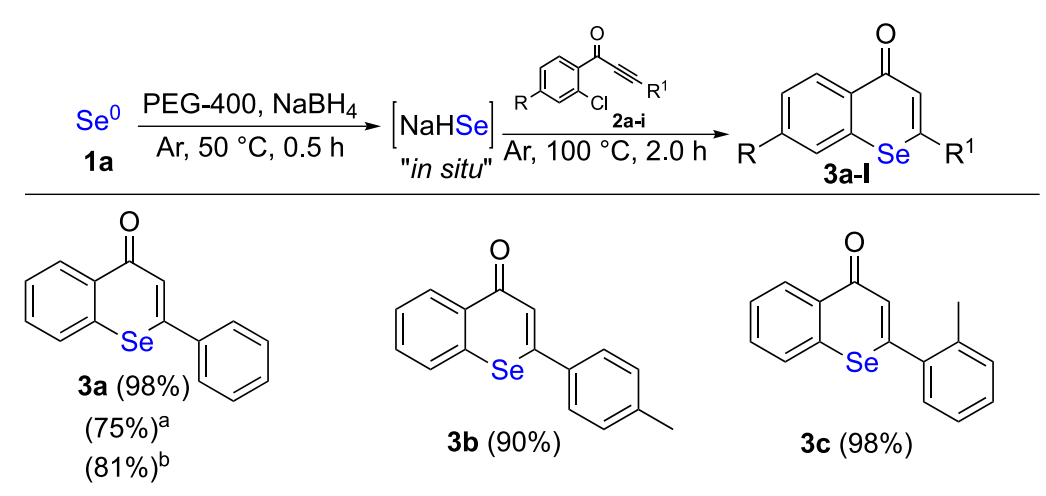<smiles>Cc1ccc(-c2cc(=O)c3ccccc3[se]2)cc1</smiles><smiles>Cc1ccccc1-c1cc(=O)c2ccccc2s1</smiles><smiles>O=c1cc(-c2ccc(Cl)cc2)[se]c2ccccc12</smiles><smiles>O=c1cc(-c2ccccc2Cl)[se]c2ccccc12</smiles><smiles>O=c1cc(-c2ccc3ccccc3c2)[se]c2ccccc12</smiles><smiles>O=c1cc(/C=C/c2ccccc2)[se]c2ccccc12</smiles><smiles>CCCCCc1cc(=O)c2ccccc2[se]1</smiles><smiles>O=c1cc(-c2ccccc2)[se]c2cc(Cl)ccc12</smiles>

Scheme 2. Substrate scope to the synthesis of Se-flavones $\mathbf{3 a}-3 \mathbf{3 i}$. Reactions was performed using $\mathrm{Se}^{0} \mathbf{1 a}(0.3 \mathrm{mmol}), \mathrm{NaBH}_{4}(0.7 \mathrm{mmol}, 2.33$ equiv in relation to selenium powder) in $2.0 \mathrm{~mL}$ of the solvent at $50{ }^{\circ} \mathrm{C}$ under argon for $0.5 \mathrm{~h}$. Then, $0.25 \mathrm{mmol}$ of 2 was added. Yields for the isolated products.

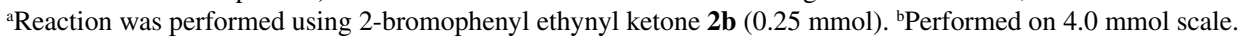


was afforded in $99 \%$ yield. Next, we explore the influence of the substituents on the 2-chlorophenyl ethynyl ketone $\mathbf{2}$ in the $\mathrm{R}^{1}$ position, firstly, containing electron-donating groups (2- $\mathrm{CH}_{3}$ and $4-\mathrm{CH}_{3}$ ), and subsequently with electronwithdrawing atoms $(2-\mathrm{Cl}$ and $4-\mathrm{Cl})$ in the aromatic ring. In both cases, substituents at the ortho position in the aromatic ring showed better results, when compared with the para position, affording the desired products $\mathbf{4 c}$ and $\mathbf{4 e}$ in 81 and $83 \%$ yield, respectively. According to the groups in the para position in the aromatic ring, the S-flavones $4 \mathbf{b}$ and $4 d$ were obtained in 75 and $62 \%$ yield, respectively. Similar to the selenium reactivity, 2-naphthyl substituent did not influence the reaction performance, leading to product $\mathbf{4 f}$ in an excellent yield of $94 \%$. To our delight, when using the compound $\mathbf{2 g}$, possessing a styryl moiety, the respective S-flavone was obtained in $61 \%$ yield and no parallel reaction in the $\mathrm{C}-\mathrm{C}$ double bond of the styrene group being observed. In addition, changing the substituent $\mathrm{R}^{1}$ to an aliphatic chain was possible, and the desired product $\mathbf{4 h}$ was obtained in $91 \%$ yield. We next evaluated the reaction of 2,4-dichlorophenyl ethynyl ketone $2 \mathbf{i}$ containing a chlorine atom in the $\mathrm{R}$ position, under optimized conditions, and the S-flavone $4 \mathbf{i}$ was obtained in a good yield. In counterpart, our attempts to synthesize the Te-flavones derivatives through the reaction between tellurium powder and compound $\mathbf{2 a}$ under the optimized conditions have been proved fruitless. In all cases, only reduction by-products derived from substrate $\mathbf{2 a}$ were observed by GC-MS analysis. ${ }^{22}$

In order to clarify the possible mechanism of the ring closure reaction, a set of control experiments were carried out (Scheme 4). Polyethylene glycols, openchain analogs of crown ethers, are well documented to have the tendency to bind with alkali cations as crown ethers. ${ }^{23}$ Our hypothesis is that the PEG-400 could be forming a crown-like structure in this reaction. For this purpose, the 2-chlorophenyl ethynyl ketone 2a was reacted with selenium powder $\mathbf{1 a}, \mathrm{NaBH}_{4}$ using ethanol as solvent (since among the tested solvents, it was able to form Se-flavone in a low yield; Table 1, entry 7) and 1.0 equiv of the 15 -crown- 5 was added in the reaction. In this condition, the desired product was obtained in $78 \%$ yield (Scheme 4, i). This result demonstrates that the presence of a crown-like species favors the formation of Se-flavone, once, in the absence of the crown ether, only $32 \%$ yield of the product was obtained (Table 1, entry 7 ). Similarly, compound 2a was reacted with selenium powder $\mathbf{1 a}, \mathrm{NaBH}_{4}$ using ethanol as solvent and 1.0 equiv of the PEG-400 was added in the reaction. Gratifyingly, the desired product was obtained in $71 \%$ yield. This result indicates a possible coordination of the PEG-400, such crown-like, to sodium atom and this complex cause the selenium anion to be more activated, favoring the formation of the Se-flavone 3a (Scheme 4, ii).

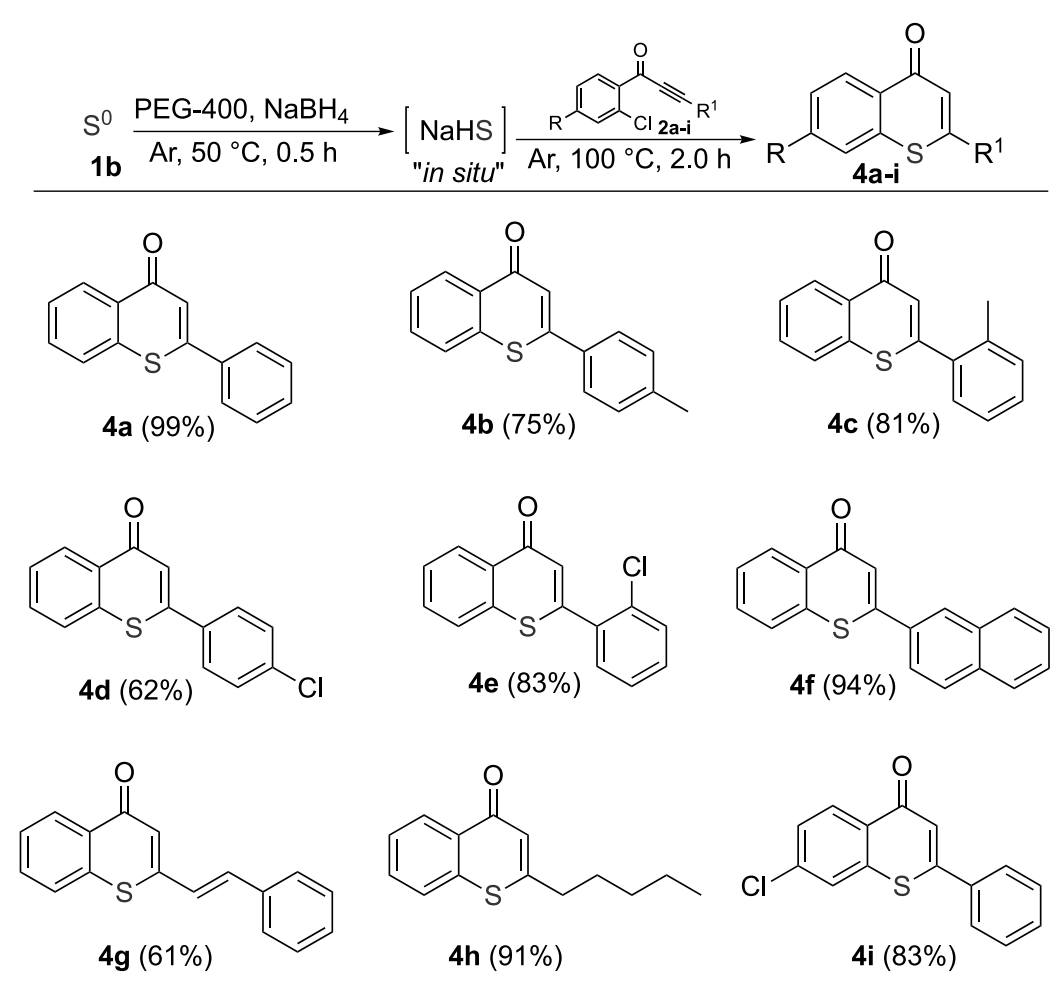

Scheme 3. Substrate scope to the synthesis of S-flavones $\mathbf{4 a - 4 i}$. Reactions was performed using $\mathrm{S}^{0} \mathbf{1 b}(0.3 \mathrm{mmol})$, NaBH $\mathrm{H}_{4}(0.7 \mathrm{mmol}, 2.33$ equiv in relation to sulfur powder) in $2.0 \mathrm{~mL}$ of the solvent at $50{ }^{\circ} \mathrm{C}$ under argon for $0.5 \mathrm{~h}$. Then, $0.25 \mathrm{mmol}$ of 2 was added. Yields for isolated products. 


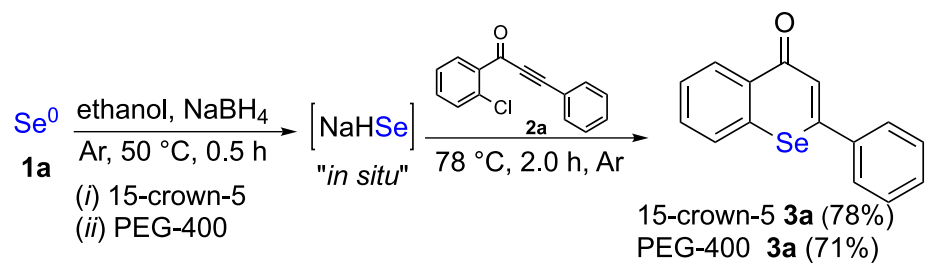

Scheme 4. Control experiments.

Our next endeavor was to identify the nucleophilic species formed by the reaction of the selenium powder with $\mathrm{NaBH}_{4}$ in PEG-400 solvent, through ${ }^{77} \mathrm{Se}$ NMR spectroscopy. In 2016, Oliveira et al. ${ }^{24}$ reported the identification of the nucleophilic selenium species from the reaction between $\mathrm{Se}^{0}$ and $\mathrm{NaBH}_{4}$ in ethanol solvent through a proton-coupled ${ }^{77} \mathrm{Se} \mathrm{NMR}$ experiment. Thus, a similar procedure was performed, and a mixture of selenium powder $1 \mathbf{a}(0.3 \mathrm{mmol})$ and $\mathrm{NaBH}_{4}(0.7 \mathrm{mmol})$ was stirred at $50{ }^{\circ} \mathrm{C}$ in PEG-400 for $0.5 \mathrm{~h}$ and then a ${ }^{77} \mathrm{Se}$ NMR spectrum was immediately recorded. It was observed in the selenium spectrum a doublet resonance at $-458.1 \mathrm{ppm}$ with a coupling constant of $16.6 \mathrm{~Hz}$, confirming the Se-H bonding (Figure 2). Oliveira et al. ${ }^{24}$ reported a similar result $(\delta=-496 \mathrm{ppm})$, corroborating the characterization of this nucleophilic selenium species. It is noteworthy that as ${ }^{15} \mathrm{~N}$ and ${ }^{17} \mathrm{O}$ NMR profile, ${ }^{25,26}$ the deshielding of the ${ }^{77} \mathrm{Se}$ chemical shift in PEG-400 solvent indicates a solvent effect, producing a less effective solvation and, consequently, obtaining a more nucleophilic selenium species. ${ }^{27}$

On the basis of the above results and reported literature, ${ }^{6}$ a plausible mechanism is proposed (Scheme 5). Firstly, the chalcogen $\mathbf{1}$ in its elemental form is reduced by $\mathrm{NaBH}_{4}$, forming the corresponding sodium hydrochalcogenide (NaHSe or NaHS). In the presence of PEG-400, which behaves as a crown ether, the in situ formation of sodium complex is favored, making the chalcogen nucleophilic species species more active. Subsequently, the nucleophilic

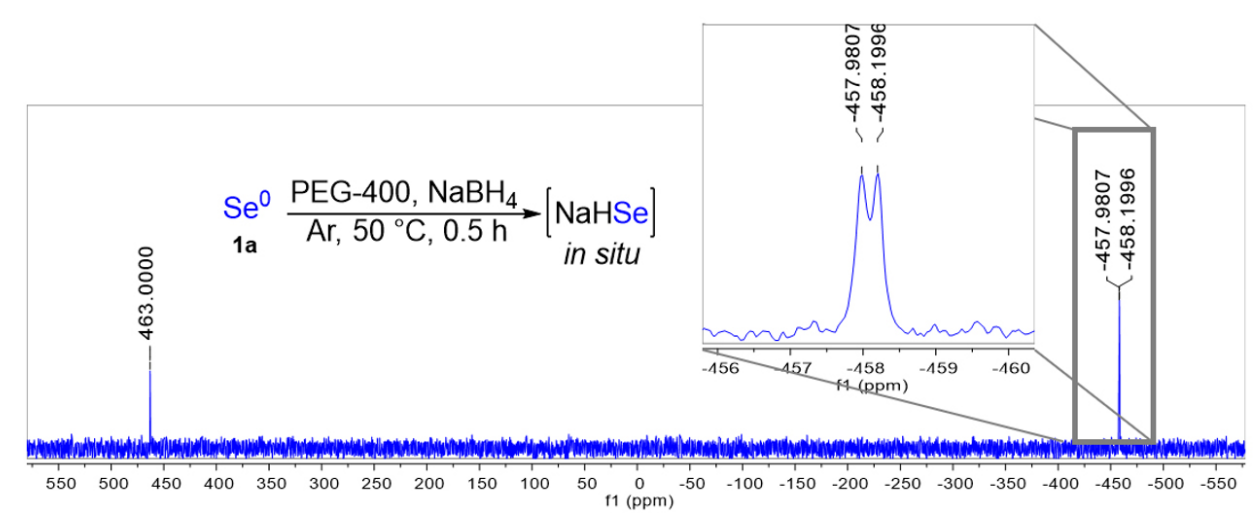

Figure 2. ${ }^{77} \mathrm{Se}$ NMR experiments to determination of the intermediate.

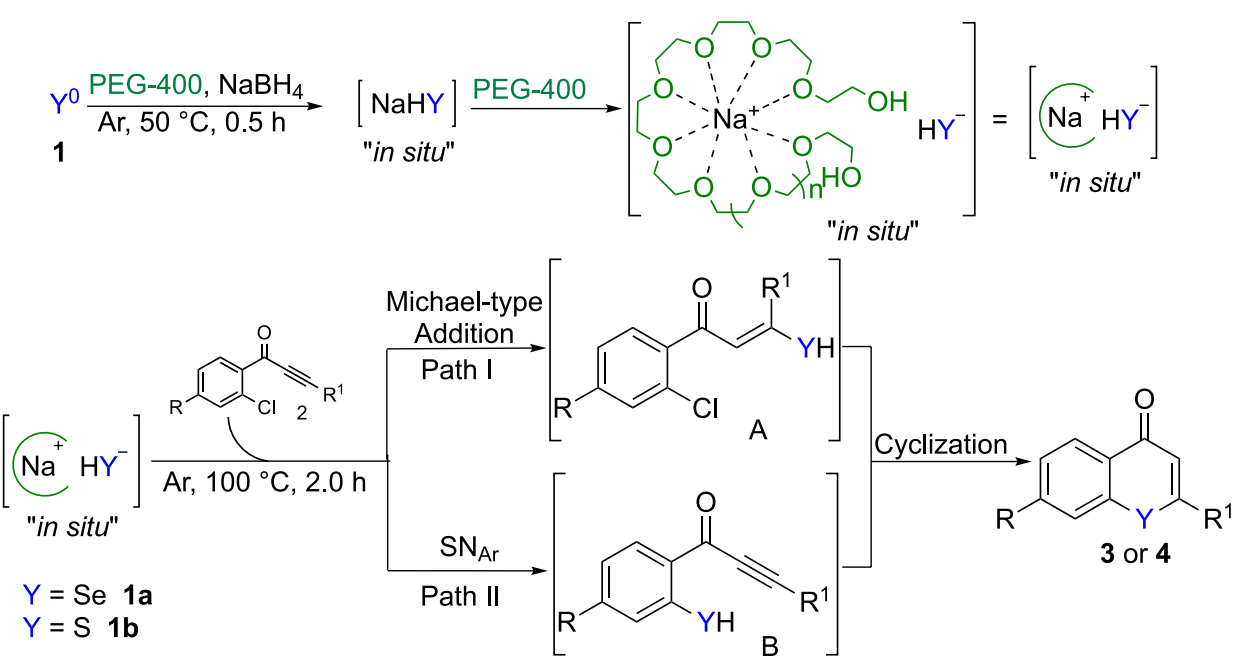

Scheme 5. Plausible mechanism. 
species is added to the carbon-carbon triple bond of the 2-chlorophenyl ethynyl ketone $\mathbf{2}$ in a Michaeltype addition, giving the intermediate $\mathbf{A}$, that after an intramolecular cyclization result in the chalcogenoflavones 3 or 4 (Scheme 5, path I). The nucleophilic species can also attack the carbon atom attached to the chlorine atom of the compound $\mathbf{2}$ in an aromatic nucleophilic substitution reaction giving the intermediate $\mathbf{B}$, that after the intramolecular cyclization results in the chalcogenoflavones 3 or 4 (Scheme 5, path II).

\section{Conclusions}

In summary, we have developed an efficient method for the synthesis of chalcogenoflavones by ring closure reaction of 2-chlorophenyl ethynyl ketones with nucleophilic chalcogen species. The nucleophiles were generated in situ using PEG-400 as solvent and $\mathrm{NaBH}_{4}$ as reduction agent. This green and environmentally friendly strategy demonstrates great compatibility with selenium and sulfur derivatives, affording a series of chalcogenoflavones in good to excellent yields. Moreover, PEG-400 proved to be crucial in this method, acting as a green solvent and mainly forming crown-like complexes. In addition, the nucleophilic species was identified by proton-coupled ${ }^{77} \mathrm{Se}$ NMR experiments, contributing to the understanding about the reactivity of this nucleophilic chalcogen species in organic synthesis.

\section{Supplementary Information}

Supplementary information is available free of charge at http://jbcs.sbq.org.br as PDF file.

\section{Acknowledgments}

This study was financed in part by the Coordenação de Aperfeiçoamento de Pessoal de Nível Superior-Brazil (CAPES) - Finance Code 001. FAPERGS (PqG 19/25510001867-3), CNPq and FINEP are acknowledged for financial support. $\mathrm{CNPq}$ is also acknowledged for fellowships to RGJ and GP.

\section{Author Contributions}

Patrick C. Nobre proposed the methodology adopted and conducted the experimental investigation; Thiago J. Peglow and Ricardo H. Bartz conducted the experimental investigation; Angelita M. Barcellos performed the formal analysis of data and visualization; Raquel G. Jacob, Márcio S. Silva, Thiago Barcellos and Gelson Perin conceptualized the experiment, funding acquisition, wrote the paper and supervision. Thiago Barcellos and Gelson Perin were responsible for project administration and coordinated the planning and execution of the study.

\section{References}

1. Santi, C.; Organoselenium Chemistry: Between Synthesis and Biochemistry; Santi, C., ed.; Bentham Science: Sharjah U. A. E., 2014; Lenardão, E. J.; Santi, C.; Sancineto, L.; New Frontiers in Organoselenium Compound; Springer: Cham, Switzerland, 2018; Jain, V. K.; Priyadarsini, K. I.; Organoselenium Compounds in Biology and Medicine Synthesis, Biological and Therapeutic Treatments; Royal Society of Chemistry: London, UK, 2017.

2. Barth, A.; Vogt, A. G.; Reis, A. S.; Pinz, M. P.; Krüger, R.; Domingues, W. B.; Alves, D.; Campos, V. F.; Pinton, S.; Paroul, N.; Wilhelm, E. A.; Luchese, C.; Mol. Neurobiol. 2019, 56, 6398; Mołchowski, J.; Kloc, K.; Lisiak, R.; Potaczek, P.; Wójtowicz, H.; Arkivoc 2007, vi, 14; Vogt, A. G.; Voss, G. T.; Oliveira, R. L.; Paltian, J. J.; Duarte, L. F. B.; Alves, D.; Jesse, C. R.; Roman, S. S.; Roehrs, J. A.; Wilhelm, E. A.; Luchese, C.; Chem.-Biol. Interact. 2018, 282, 7; Lopes, E. F.; Penteado, F.; Thurow, S.; Pinz, M.; Reis, A. S.; Wilhelm, E. A.; Luchese, C.; Barcellos, T.; Dalberto, B.; Alves, D.; Silva, M. S.; Lenardão, E. J.; J. Org. Chem. 2019, 84, 12452; Soares, A. T. G.; Junior, L. B. L. R.; Salgueiro, W. G.; dal Forno, A. H. C.; Rodrigues, C. F.; Sacramento, M.; Franco, J.; Alves, D.; Oliveira, R. P.; Pinton, S.; Ávila, D. S.; J. Trace Elem. Med. Biol. 2019, 53, 34; Couto, S. F.; Araujo, S. M.; Bortolotto, V. C.; Poetini, M. R.; Pinheiro, F. C.; Musachio, E. A. S.; Meichtry, L. B.; Sacramento, M.; Alves, D.; Novo, D. L. R.; Mesko, M. F.; Prigol, M.; J. Trace Elem. Med. Biol. 2019, 54, 232.

3. Khadem, S.; Marles, R. J.; Molecules 2012, 17, 191; Sharma, S. K.; Kumar, S.; Chand, K.; Kathuria, A.; Gupta, A.; Jain, R.; Curr. Med. Chem. 2012, 18, 3825; Gaspar, A.; Matos, M. J.; Garrido, J.; Uriarte, E.; Borges, F.; Chem. Rev. 2014, 114, 4960; Keri, R. S.; Budagumpi, S.; Pai, R. K.; Balakrishna, R. G.; Eur. J. Med. Chem. 2014, 78, 340.

4. Horvath, A.; Nussbaumer, P.; Wolff, B.; Billich, A.; J. Med. Chem. 2004, 47, 4268; Gotoda, S.; Takahashi, N.; Nakagawa, H.; Murakami, M.; Takechi, T.; Komura, T.; Uchida, T.; Takagi, Y.; Pestic. Sci. 1998, 52, 309; Zhang, D.; Ji, X.; Gao, R.; Wang, H.; Meng, S.; Zhong, Z.; Li, Y.; Jiang, J.; Li, Z.; Acta Pharm. Sin. B 2012, 2, 575; Kataoka, T.; Watanabe, S.-i.; Mori, E.; Kadomoto, R.; Tanimura, S.; Kohno, M.; Bioorg. Med. Chem. 2004, 12, 2397; Dong, J.; Zhang, Q.; Meng, Q.; Wang, Z.; Li, S.; Cui, J.; Mini-Rev. Med. Chem. 2018, 18, 1714; Choi, E. J.; Lee, J. I.; Kim, G.-H.; Int. J. Mol. Med. 2012, 29, 252; Choi, Y.-S.; Kim, D.-M.; Kim, Y.-J.; Yang, S.; Lee, K.-T.; Ryu, J. H.; Jeong, J.-H.; Int. J. Mol. Sci. 2015, 16, 29574; Holshouser, M. H.; Loeffler, 
L. J.; Hall, I. H.; J. Med. Chem. 1981, 24, 853; Nussbaumer, P.; Lehr, P.; Billich, A.; J. Med. Chem. 2002, 45, 4310.

5. Alcaide, B.; Almendros, P.; Lázaro-Milla, C.; DelgadoMartínez, P.; Chem.-Eur. J. 2018, 24, 8186; Kobayashi, K.; Kobayashi, A.; Ezaki, K. A.; Heterocycles 2012, 85, 1997; Luxen, A. J.; Christiaens, L. E. E.; Renson, M. J.; J. Organomet. Chem. 1985, 287, 81; Xu, J.; Zhang, F.; Zhang, S.; Zhang, L.; Yu, X.; Yan, J.; Song, Q.; Org. Lett. 2019, 21, 1112.

6. Fuchs, F. C.; Eller, G. A.; Holzer, W.; Molecules 2009, 14, 3814; Sangeetha, S.; Sekar, G.; Org. Lett. 2019, 21, 75; Yang, W.-R.; Choi, Y.-S.; Jeong, J.-H.; Org. Biomol. Chem. 2017, 15, 3074; Sashida, H.; Synthesis 1998, 745; Pistoia, R. P.; Back, D. F.; Zeni, G.; Eur. J. Org. Chem. 2019, 3794.

7. Shen, C.; Spannenberg, A.; Wu, X.-F.; Angew. Chem., Int. Ed. 2016, 55, 5067; Willy, B.; Frank, W.; Müller, T. J. J.; Org. Biomol. Chem. 2010, 8, 90; Zhang, F.-L.; Chen, Z.-B.; Liu, K.; Yuan, Q.; Jiang, Q.; Zhu, Y.-M.; Synlett 2018, 29, 621; Willy, B.; Müller, T. J. J.; Synlett 2009, 1255.

8. Lee, J. I.; Bull. Korean Chem. Soc. 2009, 30, 710.

9. Lee, J. I.; Kim, M. J.; Bull. Korean Chem. Soc. 2011, 32, 1383.

10. Inami, T.; Kurahashi, T.; Matsubara, S.; Org. Lett. 2014, 16, 5660.

11. Vijay, T. A. J.; Nandeesh, K. N.; Raghavendra, G. M.; Rangappa, K. S.; Mantelingu, K.; Tetrahedron Lett. 2013, 54, 6533.

12. Yang, X.; Li, S.; Liu, H.; Jiang, Y.; Fu, H.; RSC Adv. 2012, 2, 6549; Nakazumi, H.; Ueyama, T.; Kitao, T.; J. Heterocycl. Chem. 1984, 21, 193; Huang, C.-N.; Chuang, R.-R.; Kuo, P.-Y.; Yang, D.-Y.; Synlett 2008, 1825; Gu, Z.-Y.; Cao, J.-J.; Wang, S.-Y.; Ji, S.-J.; Chem. Sci. 2016, 7, 4067; Wang, D.; Sun, P.; Jia, P.; Peng, J.; Yue, Y.; Chen, C.; Synthesis 2017, 49, 4309.

13. Anastas, P.; Eghbali, N.; Chem. Soc. Rev. 2010, 39, 301; Marco, B. A.; Rechelo, B. S.; Tótoli, E. G.; Kogawa, A. C.; Salgado, H. R. N.; Saudi Pharm. J. 2019, 27, 1; Mulimani, P.; Br. Dent. J. 2017, 222, 954; Bryan, M. C.; Dunn, P. J.; Entwistle, D.; Gallou, F.; Koenig, S. G.; Hayler, J. D.; Hickey, M. R.; Hughes, S.; Kopach, M. E.; Moine, G.; Richardson, P.; Roschangar, F.; Stevenh, A.; Weiberth, F. J.; Green Chem. 2018, 20, 5082; Erythropel, H. C.; Zimmerman, J. B.; Winter, T. M.; Petitjean, L.; Melnikov, F.; Lam, C. H.; Lounsbury, A. W.; Mellor, K. E.; Janković, N. Z.; Tu, Q.; Pincus, L. N.; Falinski, M. M.; Shi, W.; Coish, P.; Plata, D. L.; Anastas, P. T.; Green Chem. 2018, 20, 1929.

14. Kerton, F. M.; Marriott, R.; Alternative Solvents for Green Chemistry, $2^{\text {nd }}$ ed.; RSC Publishing: Cambridge, UK, 2013; Reichardt C.; Welton, T.; Solvents and Solvent Effects in Organic Chemistry, $4^{\text {th }}$ ed.; Wiley-VCH: Weinheim, 2011.

15. Chen, J.; Spear, S. K.; Huddleston, J. G.; Rogers, R. D.; Green Chem. 2005, 7, 64.

16. Vafaeezadeh, M.; Hashemi, M. M.; J. Mol. Liq. 2015, 207, 73; Chandrasekhar, S.; Narsihmulu, C.; Sultana, S. S.; Reddy, N. R.;
Org. Lett. 2002, 25, 4399; Harraza, F. A.; El-Hout, S. E.; Killa, H. M.; Ibrahim, I. A.; J. Catal. 2012, 286, 184; Kadam, N. D.; Jayaram, R. V.; Curr. Catal. 2018, 7, 52; Kumar, D.; Patel, G.; Mishra, B. G.; Varma, R. S.; Tetrahedron Lett. 2008, 49, 6974.

17. Perin, G.; Alves, D.; Jacob, R. G.; Barcellos, A. M.; Soares, L. K.; Lenardão, E. L.; ChemistrySelect 2016, 2, 205; Perin, G.; Silveira, M. B.; Barcellos, A. M.; Jacob, R. G.; Alves, D.; Org. Chem. Front. 2015, 2, 1531; Stach, G.; Peglow, T. J.; Roehrs, J. A.; Penteado, F.; Barcellos, T.; Jacob, R. G.; Lenardão, E. J.; Perin, G.; Synthesis 2019, 51, 578; Borges, E. L.; Peglow, T. J.; Silva, M. S.; Jacoby, C. G.; Schneider, P. H.; Lenardão, E. J.; Jacob, R. G.; Perin, G.; New J. Chem. 2016, 40, 2321.

18. Perin, G.; Borges, E. L.; Alves, D.; Tetrahedron Lett. 2012, 53 , 2066; Perin, G.; Borges, E. L.; Rosa, P. C.; Carvalho, P. N.; Lenardão, E. J.; Tetrahedron Lett. 2013, 54, 1718; Perin, G.; Borges, E. L.; Peglow, T. J.; Lenardão, E. J.; Tetrahedron Lett. 2014, 55, 5652; Silva, P. C.; Borges, E. L.; Lima, D. B.; Jacob, R. G.; Lenardão, E. J.; Perin, G.; Silva, M. S.; Arkivoc 2016, v, 376; Perin, G.; Goulart, H. A.; Soares, L. K.; Peglow, T. J.; Schumacher, R. F.; Pinz, M. P.; Reis, A. S.; Luchese, C.; Appl. Biochem. Biotechnol. 2018, 184, 1398.

19. Perin, G.; Barcellos, A. M.; Luz, E. Q.; Borges, E. L.; Jacob, R. G.; Lenardão, E. J.; Sancineto, L.; Santi, C.; Molecules 2017, 22, 327; Peglow, T. J.; Schumacher, R. F.; Cargnelutti, R.; Reis, A. S.; Luchese, C.; Wilhelm, E. A.; Perin, G.; Tetrahedron Lett. 2017, 58, 3734.

20. Pierce, B. M.; Simpson, B. F.; Ferguson, K. H.; Whittaker, R. E.; Org. Biomol. Chem. 2018, 16, 6659; Vyas, V. K.; Knighton, R. C.; Bhanage, B. M.; Wills, M.; Org. Lett. 2018, 20, 975.

21. French, K. L.; Angel, A. J.; Williams, A. R.; Hurst, D. R.; Beam, C. F.; J. Heterocyclic. Chem. 1998, 35, 45.

22. Perin, G.; Barcellos, A. M.; Peglow, T. J.; Nobre, P. C.; Cargnelutti, R.; Lenardão, E. J.; Marini, F.; Santi, C.; RSC Adv. 2016, 6,103657

23. Li, Y.-N.; Wang, J.-L.; He, L.-N.; Tetrahedron Lett. 2011, 52, 3485; Humne, V.; Lokahnde, P.; Synth. Commun. 2014, 44, 929; Panwar, V.; Ray, S. S.; Jain, S. L.; Tetrahedron Lett. 2015, 56, 4184.

24. Oliveira, A. R. M.; Piovan, L.; Simonelli, F.; Barison, A.; Santos, M. F. C.; Mello, M. B. M.; J. Organomet. Chem. 2016, 806, 54.

25. Cusick, J.; Dance, I.; Polyhedron 1991, 10, 2629.

26. Brown, S. In NMR Crystallography; Harris, R. K.; Wasylishen, R. E.; Duer, M. J., eds.; John Wiley \& Sons Ltd.: Chichester, 2009, p. 321.

27. Daolio, A.; Scilabra, P.; Pietro, M. E. D.; Resnati, C.; Rissanen, K.; Resnati, G.; New J. Chem. 2020, 44, 20697.

Submitted: February 11, 2021 Published online: April 15, 2021 